\title{
Isolation and Characterization of Expression cDNA Clones Encoding Antigens of Onchocerca volvulus Infective Larvae
}

\author{
Thomas R. Unnasch,* Michaela Y. Gallin, Peter T. Soboslay, Klaus D. Erttmann, and Bruce M. Greene \\ Division of Geographic Medicine, Department of Medicine, and Department of Microbiology and Molecular Biology, \\ Case Western Reserve University, Cleveland, Ohio 44106
}

\begin{abstract}
The isolation of recombinant cDNA clones expressing antigens found in Onchocerca volvulus infective larvae is described. To isolate such clones, an expression cDNA library constructed from adult $O$. volvulus RNA was screened with antiserum raised against infective larvae. One clone, designated $\lambda$ RAL-1 was characterized further. The recombinant antigen produced by $\lambda$ RAL-1 stimulates proliferation of peripheral blood mononuclear cells from $O$. volvulus infected humans. ARAL-1 is derived from a 1450 bases message that encodes a protein with an apparent molecular weight of 42,000 in adult $O$. volvulus. The inserted DNA of $\lambda$ RAL-1 contains an open reading frame of $1008 \mathrm{bp}$. The amino acid sequence predicted by this open reading frame contains three repeats of the sequence KKPEDWD. The identification of clones such as $\lambda R A L-1$ will provide quantities of purified antigens sufficient to begin to study the immune response to and explore the development of immunity against the infectious form of the parasite.
\end{abstract}

\section{Introduction}

Onchocerciasis, or river blindness, is a major cause of infectious blindness in the world. In severely affected areas, as many as half of the adult males may be blinded by the disease (1), and mortality rates in such persons are increased as much as fourfold (2). Attempts to control the disease have centered on control of the vector for the parasite, the blackfly Simulium $s p$., as well as on the development of chemotherapeutic agents which may be used to eliminate the parasite from infected individuals. Unfortunately, control of the vector has proven extremely difficult. Furthermore, to date no practical chemotherapeutic agent has been identified that kills the adult form of the parasite.

Because neither vector control nor chemotherapy has proven successful in controlling the disease, the development of a vaccine against infection with the parasite has become a high priority (3). In order to develop a vaccine, it is necessary to first identify antigens that induce immunity against the parasite. In the case of onchocerciasis, however, the identification of such antigens is complicated by the fact that much of the

Address reprint requests to Dr. Unnasch, Division of Geographic Medicine, Case Western Reserve University, University Hospitals, 2074 Abington Road, Cleveland, $\mathrm{OH} 44106$.

Received for publication 10 November 1987 and in revised form 15 January 1988.

J. Clin. Invest.

(C) The American Society for Clinical Investigation, Inc.

$0021-9738 / 88 / 07 / 0262 / 08 \$ 2.00$

Volume 82, July 1988, 262-269 pathology seen during the course of the disease may be caused by the induction of the hosts immune response to certain antigens of the parasite (4). Because of this concern, it is necessary to delineate as fully as possible which parasite antigens are involved in inducing potentially beneficial as opposed to harmful aspects of the host immune response.

In order to undertake such a dissection of the immune response, large quantities of purified parasite antigens will be required. However, no animal host has been identified that can be used to provide the various stages of Onchocerca volvulus in quantity. As a consequence, the lack of parasite material has posed a major impediment to immunological studies. This problem has been particularly acute for the study of the infectious form of the parasite, the third stage larva, or L3. Antigens from this stage may be especially important, since it has been shown in other filarial infections that irradiated infective larvae can induce protective immunity $(5,6)$.

To circumvent the problem of the lack of parasite material, we have undertaken an alternative strategy to simply isolating antigens directly from the limited amounts of parasite material that are available. This is based upon the identification of cDNA clones that express antigens associated with $O$. volvulus infective larvae. To identify such clones, we have used antisera produced by introducing living infective larvae into a nonpermissive host to screen an $O$. volvulus expression cDNA library. Using this approach, six clones have been identified. One clone, designated $\lambda$ RAL-1, a member of the class that appears to react most strongly with the immune sera, was chosen for detailed characterization. Based upon immunoaffinity purified antibody probing of crude $O$. volvulus protein extracts, the mRNA that gave rise to $\lambda$ RAL-1 appeared to encode a protein with an apparent molecular weight of 42,000 in vivo. The recombinant antigen produced by this clone stimulated $T$ cells from infected individuals to proliferate, suggesting that the native antigen related to $\lambda R A L-1$ has relevance in natural infections. Sequence analysis of the cDNA insert of $\lambda$ RAL-1 revealed the presence of a single long open reading frame, which encodes three repeats of the polypeptide sequence KKPEDWD. The isolation and characterization of $\lambda$ RAL-1 demonstrates that it will be possible to use clones expressing antigens found in $O$. volvulus infective larvae to examine the role that such antigens may play in the development of a protective immune response against the parasite.

\section{Methods}

Isolation of $O$. volvulus $R N A$ and DNA. RNA was prepared from freshly excised nodules obtained from people infected with $O$. volvulus residing in Grand Bassa county, Liberia. The nodules were carefully trimmed to remove as much host tissue as possible. The parasite material was immediately homogenized in a solution of $6 \mathrm{M}$ guanidium thiocyanate, $5 \mathrm{mM}$ sodium citrate (pH 7.0), $100 \mathrm{mM} \beta$-mercaptoethanol and $0.5 \%$ sarkosyl, using a mechanical tissue grinder. The extracts were frozen at $-20^{\circ} \mathrm{C}$, and transported to the United States where 
RNA was isolated by centrifugation through a $\mathrm{CsCl}$ cushion, as described by Glisin et al. (7). Between 15 and $30 \mu \mathrm{g}$ of RNA was obtained per adult female worm.

$O$. volvulus genomic DNA was prepared from adult worms that were freed from surrounding host tissue using the collagenase digestion protocol described by Schulz-Key (8) and stored in liquid nitrogen. The DNA was prepared using the method described by Shah et al. (9). Between 3 and $10 \mu \mathrm{g}$ of DNA was obtained per adult female worm. Each parasite DNA preparation was tested for the presence of contaminating host cell DNA by hybridization with a variety of probes for different human repeated genes. No contaminating host cell DNA was detected in any of the preparations used in the experiments described below.

In vitro translation of parasite $R N A$ and immunoprecipitation of the translated polypeptides. Total RNA isolated from adult $O$. volvulus and Dirofilaria immitis was translated in vitro in rabbit reticulocyte lysates (Promega Biotech, Madison, WI), using the protocol provided by the manufacturer. Rabbit antisera were prepared for use in immunoprecipitation of the translation products by heating to $60^{\circ} \mathrm{C}$ for 20 min. Immunoglobulins were precipitated by addition of ammonium sulfate to a final saturation of $\mathbf{4 0 \%}$. Precipitated protein was resuspended in $25 \mathrm{mM}$ Tris- $\mathrm{HCl}$ (pH 7.5), $100 \mathrm{mM} \mathrm{NaCl}$, and dialyzed against the same buffer overnight at $4^{\circ} \mathrm{C}$. Aliquots of the in vitro translation products and the purified antisera were mixed together and incubated overnight at $4^{\circ} \mathrm{C}$. The antibody-antigen complexes were then bound to Protein A Sepharose beads (Pharmacia, Uppsala, Sweden). The beads were washed repeatedly in a solution of $50 \mathrm{mM}$ Tris (pH 6.9), $150 \mathrm{mM} \mathrm{NaCl}, 5 \mathrm{mM}$ EDTA, $1 \%$ NP-40, $1 \%$ sodium deoxycholate, $0.2 \%$ SDS, and $0.5 \%$ bovine serum albumin. The beads were resuspended in a solution of $62.5 \mathrm{mM}$ Tris-glycine (pH 6.8), 3\% SDS, $0.5 \%$ 2-mercaptoethanol, and $10 \%$ glycerol, and heated for $3 \mathrm{~min}$ at $100^{\circ} \mathrm{C}$. Freed polypeptides were separated on a $10 \%$ polyacrylamide gel, prepared as described by Laemmli (10). Following electrophoresis, the gel was treated with the autoradiography enhancer Enlightening (New England Nuclear), dried and subjected to autoradiography.

Preparation of $O$. volvulus expression $C D N A$ library. $O$. volvulus RNA, prepared as described above, was passed over an oligo-dT cellulose column, and poly $\mathrm{A}^{+}$RNA eluted from the column as described by Maniatis et al. (11). The poly $\mathrm{A}^{+}$fraction from the column was then used to prepare first and second strand CDNA, using the RNAse $H$ protocol of Gubler and Hoffman (12). The CDNA was treated with Eco RI methylase (New England Biolabs, Beverly, MA), using the conditions described by the manufacturer. Following methylation, synthetic Eco RI linkers (Collaborative Research, Lexington, MA), phosphorylated as described by the manufacturer, were ligated to the ends of the cDNA. The preparation was then digested with Eco RI, and the freed linkers removed by chromatography on Bio-Gel P-30 (Bio Rad Laboratories, Richmond, CA). The cDNA was then ligated to $\lambda g t 11$ arms (Stratagene, San Diego, CA). The resulting concatemers were packaged using Gigapack packaging extracts (Stratagene), using the protocol provided by the manufacturer. The library produced in this manner contained $\sim 500,000$ independent recombinant clones.

Preparation of $O$. volvulus antisera. To prepare antisera against $O$. volvulus infective larvae, living $L 3 s$ were isolated by dissection from experimentally infected flies which had been maintained as described by Ham and Banya (13). A total of 164 living larvae suspended in RPMI tissue culture medium without adjuvant were injected subcutaneously into a New Zealand white rabbit. At one-week intervals following the first inoculation, the rabbit received two booster injections of $\sim 100$ living larvae. A final boost of 229 living infective larvae was administered five weeks after the initial innoculation. Eight weeks after the final injection of infective larvae, the rabbit was subjected to a detailed postmortum examination. No evidence of developing parasites was found.

Antiserum against adult $O$. volvulus was prepared by homogenizing collagenase freed adult parasites in sterile saline in a Ten-Broeck glassglass homogenizer. The extract was mixed 1:1 (vol/vol) with Freund's complete adjuvant, and used to immunize a New Zealand white rab- bit. After the initial immunization, the rabbit was boosted twice at weekly intervals with the same antigen-adjuvant mixture.

Antibody screening of the cDNA library. Portions of the cDNA library were plated at a density of $\sim 35,000$ plaque forming units per $135 \mathrm{~mm}$ Petri dish, and the plaques induced to produce fusion proteins according to the protocol of Huynh et al. (14). Duplicate filters prepared from each plate were incubated overnight at room temperature with the appropriate antiserum. Antisera used in the screening procedure were diluted $1 / 20$ in a solution of $20 \mathrm{mM}$ Tris- $\mathrm{HCl}(\mathrm{pH} 7.5), 0.5$ $\mathrm{M} \mathrm{NaCl}$, and 3\% BSA (TBS/BSA). Antibodies reacting with Escherichia coli antigens were removed prior to use by the protocol of Huynh et al. (14). Following incubation with the primary antiserum, the filters were washed in TBS/BSA, and exposed to Protein-A conjugated to horseradish peroxidase (Bio-Rad Laboratories, Richmond, CA) for $2 \mathrm{~h}$ at room temperature. Positive signals were identified by development in a solution containing $66 \mathrm{mM}$ Tris- $\mathrm{HCl},(\mathrm{pH} 7.5), 20 \%$ methanol, $0.06 \%$ 4-chloro-1-napthol, and $0.02 \%$ hydrogen peroxide.

Lymphocyte blastogenesis assays. PBMCs were isolated by FicollHypaque gradient centrifugation of fresh heparinized blood, obtained from $O$. volvulus infected individuals living in Liberia. The cells were suspended in a solution containing 40\% RPMI 1640 tissue culture medium, $40 \%$ DMSO, and $20 \%$ heat inactivated FCS, and frozen in liquid nitrogen. For the blastogenesis assays, the cells were thawed, washed twice in RPMI 1640 tissue culture medium and resuspended in RPMI 1640 containing $2 \mathrm{mM}$ L-glutamine, $100 \mathrm{U} / \mathrm{ml}$ penicillin, 100 $\mathrm{U} / \mathrm{ml}$ streptomycin, and $10 \%$ heat inactivated pooled human serum. The cells were then plated at a concentration of $10^{6}$ cells $/ \mathrm{ml}$ together with extracts of induced lysogen cultures of $\lambda R A L-1$. Extracts were prepared as described by Huynh et al. (14), dialyzed against PBS, and filtered through a $0.45-\mu \mathrm{m}$ filter before use. Extracts were used in a final concentration of $15 \mu \mathrm{g} / \mathrm{ml}$ of protein. Cultures were incubated for $78 \mathrm{~h}$ at $37^{\circ} \mathrm{C}$ in an atmosphere containing $5 \% \mathrm{CO}_{2}$. At this point, $1 \mu \mathrm{Ci}$ of $\left[{ }^{3} \mathrm{H}\right]$ thymidine (Amersham, Arlington Heights, IL) was added to the cultures, and the incubation continued for an additional $18 \mathrm{~h}$. Cells were harvested on filter paper, and $\left[{ }^{3} \mathrm{H}\right]$ thymidine incorporation assessed by counting the samples in a liquid scintillation counter. Results were expressed as the mean counts per minute of four culture wells \pm SEM.

Affinity purification of antibodies reactive with the recombinant antigen produced by $\lambda R A L-1$. Lysates of induced lysogen cultures of $\lambda g t 11$ and $\lambda$ RAL-1 were prepared according to the protocol of Huynh et al. (14). Protein from these lysates was coupled to cyanogen bromide activated Sepharose CL-6B (Pharmacia), using the protocol provided by the manufacturer. An aliquot of the antiserum raised against adult $O$. volvulus was diluted $1 / 20$ in TBS/BSA. The antibody solution was then preabsorbed to remove antibodies that reacted with $E$. coli antigens following the protocol of Huynh et al. (14). The antiserum preparation was then passed through a column containing the gel to which the $\lambda g t 11$ protein had been bound. The eluate was collected from this column, and passed over a column containing the gel to which the ARAL-1 lysate protein had been attached. Both columns were washed with a solution of $50 \mathrm{mM}$ Tris- $\mathrm{HCl}(\mathrm{pH} 7.5) .0 .5 \mathrm{M} \mathrm{NaCl}$, and bound antibodies eluted with a solution containing $0.2 \mathrm{M}$ Tris ( $\mathrm{pH} 7.5)$ and 8 $\mathrm{M}$ urea. The eluate was adjusted to $3 \%(\mathrm{wt} / \mathrm{vol}) \mathrm{BSA}$ and dialyzed against TBS.

$D N A$ sequencing. Isolated cDNA insert was subcloned into the plasmid vector pUC 13, and clones containing the inserted DNA in opposite orientations identified. Plasmids containing the inserted DNA in opposite orientations were linearized by digestion with the restriction enzyme Hind III, and nested deletions prepared using the exonuclease $\mathrm{Bal}$ 31. The deleted insert DNA was liberated by digestion with Eco R1 and purified from an agarose gel as described by Vogelstein and Gillespie (15). The purified deleted insert DNA was then directionally cloned into the single stranded bacteriophage vector M13 Mp18. Clones containing deletions whose ends differed by $\sim 200$ bp in length were isolated, and single stranded phage DNA prepared as described by Schreier and Cortese (16). This DNA was then sequenced using the dideoxynucleotide termination method (17). 


\section{Results}

Immunoprecipitation of in vitro translation products of $O$. volvulus $R N A$. To examine the ability of the antisera raised against the adult and larval stages of $O$. volvulus to recognize protein epitopes, adult RNA from both $O$. volvulus and $D$. immitis was translated in vitro. In both cases, this procedure resulted in the production of $>100$ polypeptides, the largest of which had an apparent size of greater than 100,000 D (data not shown). These polypeptides were then immunoprecipated with antisera raised against the adult and larval stages of $O$. volvulus. The results of this experiment are shown in Fig. 1. The antiserum raised against the adult form of the parasite recognized greater than twenty different sized polypeptides (lane $A$, set $\mathrm{O}$ ). For most of these polypeptides, polypeptides of similar molecular weight were also recognized in the in vitro translation products of $D$. immitis (compare lanes labeled $A$ ). Some of the polypeptides recognized by the adult antiserum did appear to be specific for $O$. volvulus, however. The most prominent of these was a polypeptide of $\sim 20,000 \mathrm{D}$ (lane $A$, set 0 ).

In contrast to the antiserum raised against the adult form of the parasite, the antiserum raised against the larval form recognized only six different polypeptides (lane $L$, set $O$ ). These polypeptides were a subset of those recognized by the adult antiserum (compare lanes $A$ and $L$, set $\mathrm{O}$ ). These results demonstrated that both the antiserum against both the adult and larval forms of the parasite were capable of recognizing protein epitopes present in the absence of any polysaccharide moities.

Isolation of $c D N A$ clones expressing epitopes found in $O$. volvulus infective larvae. To identify clones expressing epitopes found in infective larvae, a portion of the cDNA library de-

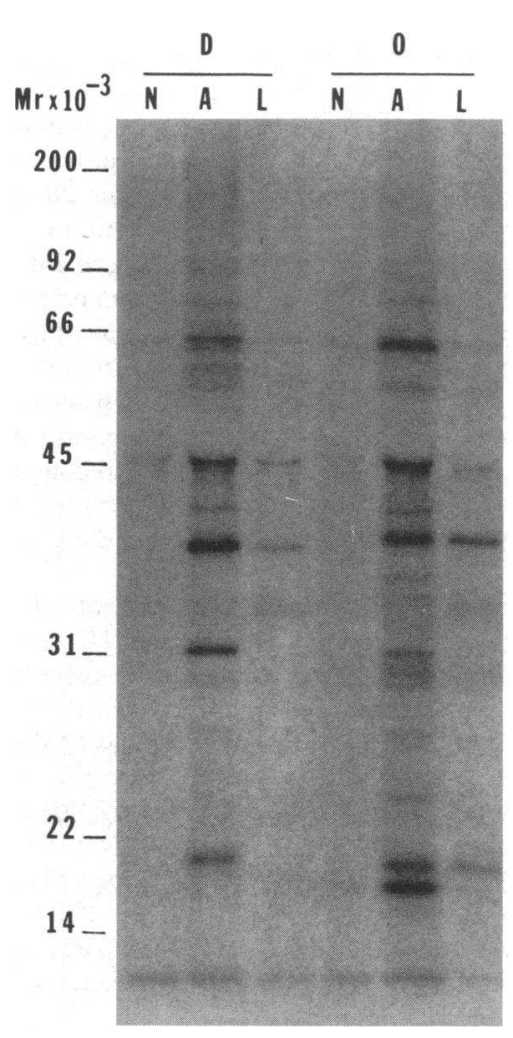

Figure 1. Immunoprecipitation of in vitro translation products of $O$. volvulus RNA. RNA from $D$. immitis and $O$. volvulus was translated in vitro and the translation products immunoprecipitated as described in Methods. The set of lanes labeled $D$ are the immunoprecipitated in vitro translation products of $D$. immitis RNA, while the set of lanes labeled $O$ are the immunoprecipitated in vitro translation products of $O$. volvulus RNA. In each of the two sets of lanes, $N$, precipitation with preimmune rabbit serum, $A$, precipitation with rabbit antiserum raised against adult $O$. volvulus, and $L$, precipitation with rabbit antiserum raised against $O$. volvulus L3s. scribed in Methods, representing $\sim 200,000$ independent recombinant clones, was screened with the larval antiserum. From this initial screen, six clones were purified which reacted reproducibly with the larval antiserum. When the inserted cDNA in each of these clones was tested for their ability to hybridize to the inserted cDNA in the other clones, it was determined that these clones could be divided into two classes. Plaque lifts were prepared from representatives from each of these classes, and the filters probed with the larval and adult antisera. As shown in Fig. 2, rows $C$ through $E$, all of the clones that reacted with the larval antiserum also reacted with the adult antiserum. However, it was possible to isolate clones with the adult antiserum that did not react with the larval antiserum (Fig. 2, row B). A member of the class of clones that reacted the most strongly with the larval antiserum (Fig. 2, rows $C$ and $D$ ), designated $\lambda$ RAL-1, was chosen for further analysis.

Induction of lymphocyte blastogenesis in cells from $O$. volvulus infected individuals by induced lysogen cultures of $\lambda R A L-1$. As a first step towards examining if the native protein related to the recombinant antigen produced by $\lambda$ RAL- 1 plays any role in the development of an immune response against the parasite in natural infections, the ability of lysates containing the recombinant antigen to stimulate proliferation of PBMCs isolated from infected individuals was examined. As shown in Table I, lysates containing the recombinant protein stimulated proliferation of PBMCs from three infected individuals to a greater extent than did control lysates. The difference in the level of stimulation between the control and recombinant lysates was significant, as judged by Student's paired $t$ test $(P<0.01)$. In contrast, when PBMCs from uninfected individuals residing in the same area were used in the assay, no significant difference between the two lysate preparations was seen (Table I).

Expression of the inserted $c D N A$ of $\lambda R A L-1$ in the plasmid vector $p U C$ 13. Initial experiments with extracts prepared from induced lysogen cultures of $\lambda$ RAL- 1 suggested that the clone
N

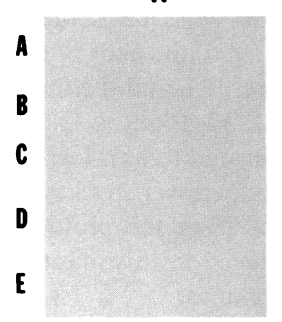

A

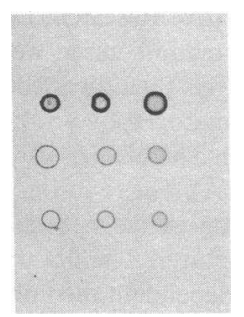

$\mathbf{l}$

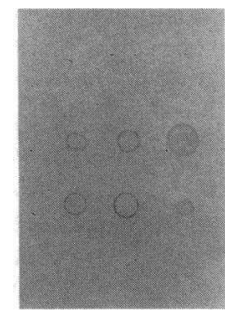

Figure 2. Identification of cDNA clones expressing antigens recognized by rabbit antisera raised against $O$. volvulus. Aliquots of plate lysates of plaque purified immunoreactive clones were spotted in a grid pattern on a lawn of $E$. coli strain Y1090. Lifts of the plaques were then prepared as described in Methods, and the filters exposed to different antisera. Positive signals were developed as described in Methods. $N$, filter exposed to preimmune rabbit serum, $A$, filter exposed to rabbit antiserum raised against adult $O$. volvulus, and $L$, filter exposed to rabbit antiserum raised against $O$. volvulus L3s. In each panel, row $A$, the vector bacteriophage $\lambda$ gt 11 , row $B$, clone $\lambda$ RAA-1, which was isolated with the anti- $O$. volvulus adult antiserum, rows $C$ and $D$, two independent isolates of the class of clones that are related to $\lambda \mathrm{RAL}-1$ by hybridization analysis, and row $E$, an example of an immunoreactive clone which is unrelated to $\lambda$ RAL-1 by hybridization analysis. 
Table I. Lysates of Cultures Containing $\lambda R A L-1$ Stimulate Proliferation of PBMCs from Infected Individuals

\begin{tabular}{|c|c|c|c|}
\hline \multirow[b]{2}{*}{ Cell source } & \multicolumn{3}{|c|}{ Culture additions } \\
\hline & Buffer & Agt 11 lysate & $\lambda$ RAL-1 lysate \\
\hline \multicolumn{4}{|l|}{ Patients } \\
\hline A & $379 \pm 74$ & $472 \pm 60$ & $1,114 \pm 322$ \\
\hline B & $296 \pm 23$ & $461 \pm 10$ & $878 \pm 48$ \\
\hline $\mathrm{C}$ & $180 \pm 10$ & $543 \pm 151$ & $1,165 \pm 6$ \\
\hline \multicolumn{4}{|l|}{ Controls } \\
\hline A & $317 \pm 54$ & $725 \pm 187$ & $799 \pm 112$ \\
\hline B & $390 \pm 42$ & $404 \pm 34$ & $319 \pm 46$ \\
\hline $\mathrm{C}$ & $425 \pm 29$ & $365 \pm 65$ & $421 \pm 66$ \\
\hline
\end{tabular}

Blastogenesis assays were performed on PBMCs isolated from three patients with $O$. volvulus infections, as well as cells from three control individuals residing in the same area, as described in Methods. The control individuals were free of clinical symptoms of onchocerciasis, contained no detectable skin microfilariae, and did not have an antibody response to $O$. volvulus, as judged by Western blot analysis with crude $O$. volvulus adult worm antigen preparations. Results are expressed as the mean counts per minute of four independent determinations $\mathbf{S E M}$.

was not producing a stable recombinant polypeptide. However, when the inserted DNA was subcloned into the plasmid vector pUC 13 a stable product with an apparent molecular weight of 38,000 was produced (Fig. 3). A doublet of smaller products recognized by the antiserum was also produced from this preparation. The relative amount of these products varied from preparation to preparation, suggesting that the smaller polypeptides were degradation products of the larger polypeptide. As is shown in Fig. 3, the production of this polypeptide was dependent on the orientation in which the CDNA insert was cloned into the vector plasmid, suggesting that its production was being controlled by the $\beta$-galactosidase gene of pUC 13.

Identification of the parasite antigen produced by the $m R N A$ that gave rise to $\lambda R A L-1$. To characterize the native antigen encoded by the mRNA that gave rise to $\lambda R A L-1$, antibodies reacting with the recombinant protein were affinity purified as described in Methods. These antibodies were used to probe Western blots of adult $O$. volvulus proteins. The affinity purified antibodies recognized a single band of an apparent molecular weight of 42,000 (Fig. 4, lane $L$ ). No proteins were recognized by antibodies affinity purified using immobilized proteins prepared from a control $\lambda$ gt 11 lysate (Fig. 4, lane $G$ ).

Organization and expression of the gene corresponding to $\lambda R A L-1$. To determine the size of the message that gave rise to $\lambda$ RAL-1, a Northern blot prepared from adult $O$. volvulus ploy $\mathrm{A}^{+} \mathrm{RNA}$ was probed with purified $\lambda \mathrm{RAL}-1$ insert, which had been radioactively labeled by nick translation. As Fig. 5, lane O demonstrates, the inserted DNA from $\lambda$ RAL-1 hybridized to a single message of 1450 bases in size. No hybridization was detected to RNA isolated from uninfected human cells (Fig. 5, lane $\mathrm{H}$ ). The results presented in Fig. 5 also suggest that an identical message is not present in mRNA prepared from the intestinal helminthic parasite Ascaris suum. However, in overexposed autoradiographs of the blot, a small amount of hybridization to a band of 1650 bases is seen in A. suum RNA.

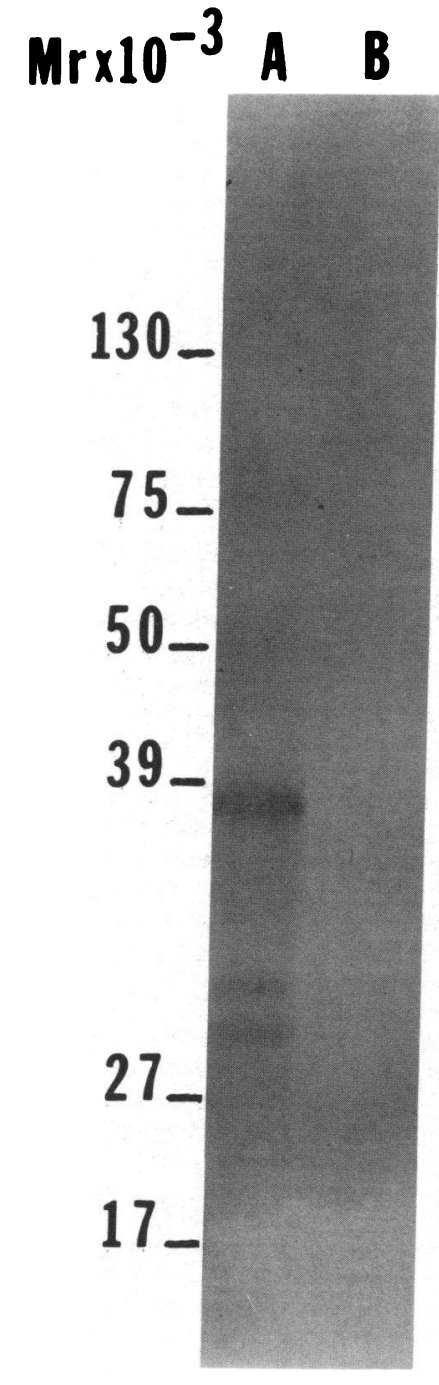

Figure 3. The inserted DNA of $\lambda$ RAL-1 encodes an antigen with an apparent molecular weight of 38,000 when inserted into the plasmid vector pUC 13. Cultures of $E$. coli containing pUC 13 plasmids into which the insert of $\lambda$ RAL-1 had been cloned in opposite orientations were induced by the addition of isopropyl $\beta$-Dgalactopyranoside to a final concentration of $10 \mathrm{mM}$. Crude lysates of the cultures were prepared, and used to prepare a Western blot, as described by Towbin et al (18). The blot was exposed to rabbit antiserum raised against adult $O$. volvulus, and developed with Protein-A conjugated with horseradish peroxidase, as described in Methods. Lane $A$, $\lambda$ RAL-1 insert cloned into pUC 13 in the plus orientation, and lane $B, \lambda R A L-1$ insert cloned into pUC 13 in the minus orientation.

The radiolabeled insert from $\lambda$ RAL- 1 was also used to probe a Southern blot prepared with both $O$. volvulus and uninfected human placental DNA. Two restriction enzymes that did not cut within the insert of $\lambda$ RAL-1 were used to prepare the blot. As is shown in Fig. 6, the insert from $\lambda$ RAL-1 hybridized to a single band in $O$. volvulus genomic DNA that had been digested with either Hind III or Eco RI. No hybridization was detected to uninfected human genomic DNA, demonstrating that the clone $\lambda$ RAL-1 was of parasite origin.

$D N A$ sequence analysis of $\lambda R A L-1$. To determine if the inserted DNA of $\lambda$ RAL-1 was related to any previously described protein or nucleic acid sequence, the DNA sequence of the inserted DNA was determined. The sequencing strategy and the complete DNA sequence of the inserted DNA is shown in Fig. 7. The insert of $\lambda$ RAL-1 was found to be $1076 \mathrm{bp}$ in length. Of these $1076 \mathrm{bp}$, only $12 \mathrm{bp}$ appear to be derived from the poly A tract at the $3^{\prime}$ end of the message. The sequence contains one large open reading frame, which extends from bases $1-1008$. This open reading frame is in frame with the $\beta$-galactosidase gene of pUC 13 (data not shown). Overall, the predicted molecular weight of a putative fusion protein produced by this open reading frame was 41,454 . Screening of the complete Genbank nucleic acid and NBRF protein data banks revealed no sequences that contained significant homol- 


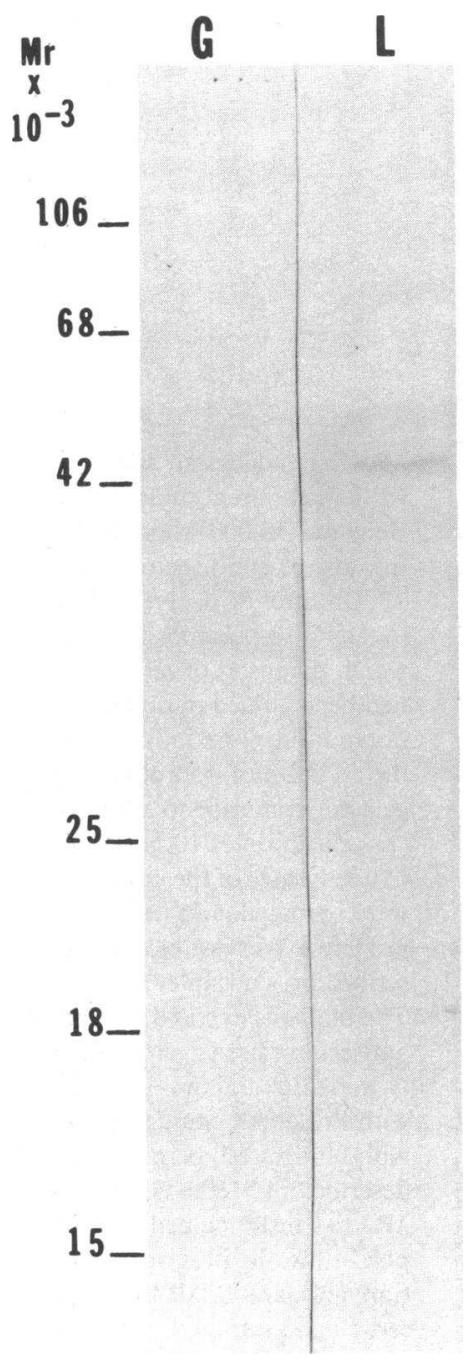

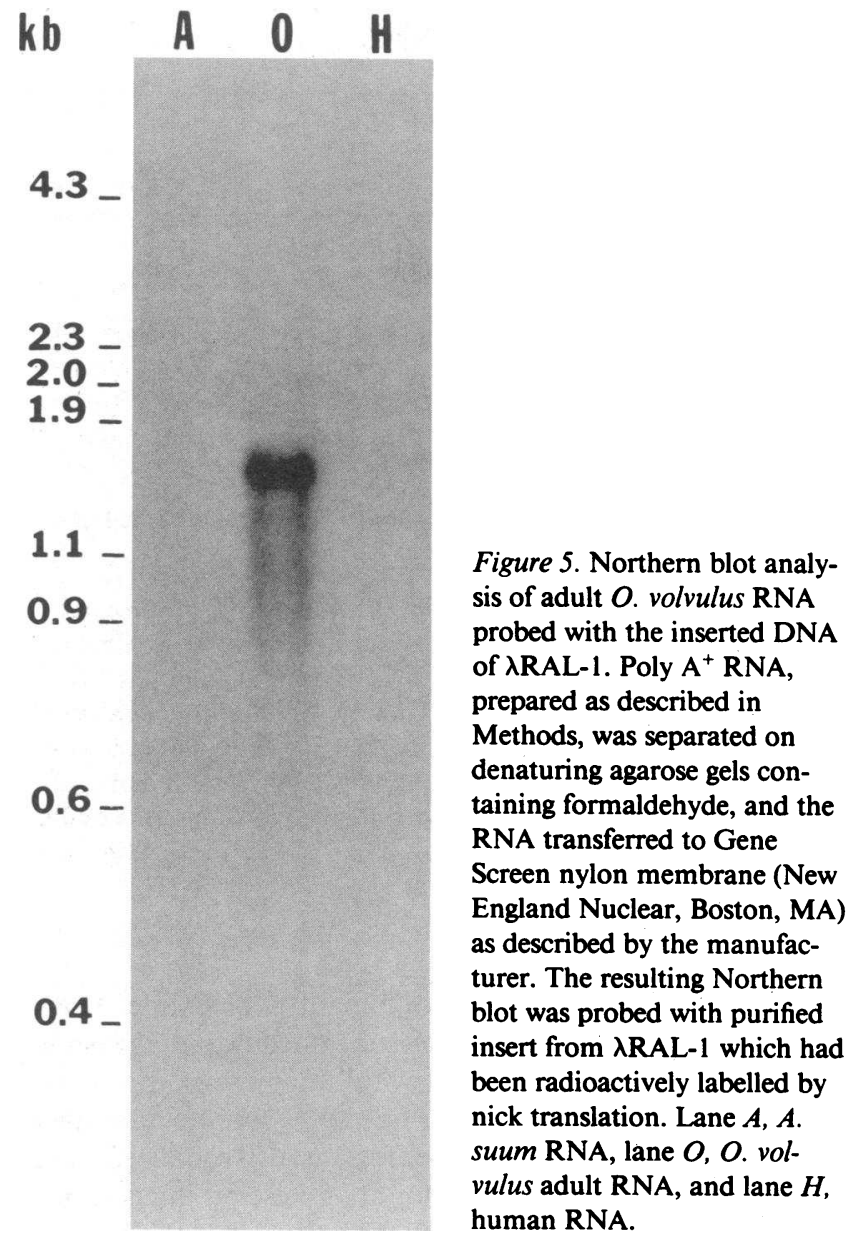

Figure 4. Identification of the native parasite antigen represented by $\lambda$ RAL- 1 . Adult $O$. volvulus were homogenized in a solution of $50 \mathrm{mM}$ Tris- $\mathrm{HCl}$ (pH 7.5), 1 mM EDTA, 0.1\% SDS, 2 $\mathrm{mM}$ iodoacetamide, $2 \mathrm{mM}$ PMSF, 2 mM TLCK, 2 mM TPCK and $2 \mu \mathrm{g} / \mathrm{ml}$ leupeptin. The homogenate was subjected to centrifugation at $81,000 \mathrm{~g}$ for $1 \mathrm{~h}$ at $4^{\circ} \mathrm{C}$. Aliquots of the supernatent containing $150 \mu \mathrm{g}$ of protein were used to prepare a Western blot as described by Towbin et al. (18). The blot was exposed to affinity purified antibodies, prepared as described in Methods, and the blot developed with Protein A conjugated with horseradish peroxidase, as described in Methods. $G$, antibodies eluted from the column containing immobilized $\lambda g t 11$ proteins, and $L$, antibodies eluted from the column containing immobilized XRAL-1 proteins.

ogies to the inserted DNA of $\lambda$ RAL-1, or to the polypeptide predicted by the long open reading frame.

As is shown in Fig. 7, the long open reading frame encoded a polypeptide that contained three repeats of the amino acid sequence KKPEDWD. The three repeats are separated from one another by two unrelated stretches of 10 amino acids each. Comparison of the DNA sequences of the repeats demonstrated that the exact homology of the repeats is not conserved at the nucleic acid level. Relative hydrophilicity plots using the algorithm of Hopp and Woods (20) indicated that the region containing the repeated sequence is highly hydrophilic (data not shown).

Following the long open reading frame, the inserted cDNA contained a putative untranslated region of $56 \mathrm{bp}$. As is shown in Fig. 7, this region contained two stretches of the poly A tract addition signal sequence AATAAA (21) within 30 bases of the start of the poly A tract at the $3^{\prime}$ end of the message.

\section{Discussion}

A central problem in the identification of immunologically important antigens of many human parasites is the difficulty in obtaining sufficient quantities of the relevant antigens to test for their biological effects. This has been a particular of $\lambda$ RAL-1. Poly $A^{+}$RNA, prepared as described in Methods, was separated on denaturing agarose gels containing formaldehyde, and the RA transferred to Gene suum RNA, lane $O, O$. volus adult RNA, and lane $H$ human RNA.

problem in the study of onchocerciasis, where there is no readily available animal host for the adult form of the parasite, which necessitates the isolation of parasite material from clinical samples. It has been even more difficult to study the antigens of the infectious form of the parasite, the infective larvae or L3. This is due to the fact that the infective larvae must be obtained by dissection of infected blackflies, which are extremely difficult to obtain. For this reason, it has not previously been possible to study the antigens of the $O$. volvulus L3. The experiments described above demonstrate that it is possible to isolate clones which express antigens of $O$. volvulus infective larvae from an expression cDNA library prepared from adult mRNA. Because the library was prepared from the adult form of the parasite, it is not surprising that all of the clones isolated to date produce antigens which are recognized by antisera raised against the adult as well as the larval form of the parasite. It is likely that any additional clones isolated using these reagents will also be recognized by the adult antiserum, although it is possible that some clones isolated in this way may be expressed at different levels in adults and infective larvae. Although these epitopes will be expressed in these two stages of the parasite's lifecycle, whether or not they may be expressed in other stages of the life cycle, such as microfilariae, cannot be predicted based on present knowledge.

Since living larvae were used in the immunization proto$\mathrm{col}$, it is possible that some of the antigens recognized by the antilarval antiserum may in fact be expressed by parasites that 


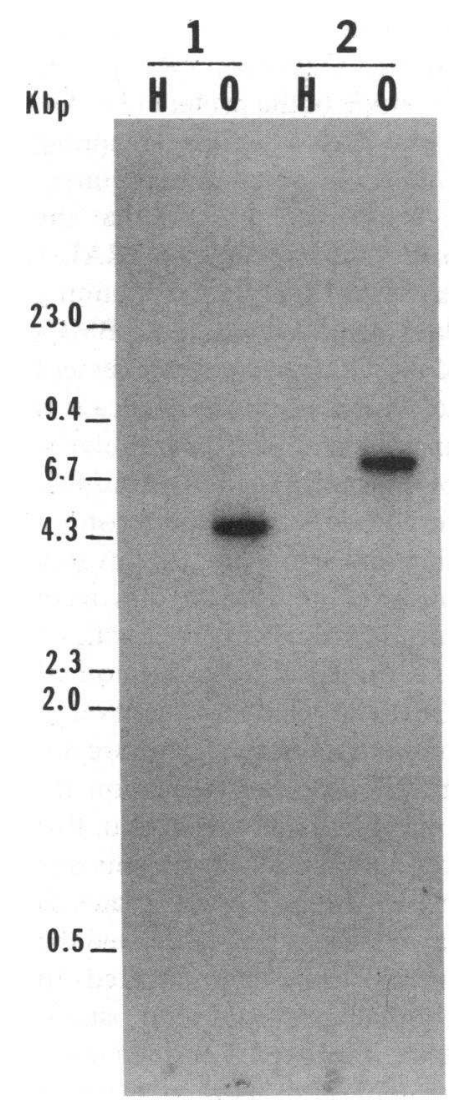

Figure 6. Southern blot analysis of genomic DNA probed with the inserted DNA of $\lambda$ RAL-1. Aliquots of human and $O$. volvulus genomic DNA digested with the restriction enzymes Eco RI and Hind III were used to prepare a Southern blot as described by Southern (19). The blot was probed with the purified insert from $\lambda$ RAL-1 that had been radioactively labeled by nick translation. The set of lanes labeled " 1 " includes the DNA samples digested with Hind III, and the set labeled "2" includes the samples digested with Eco RI. In each set, $H$, human DNA and $O, O$. volvulus DNA.

had undergone limited development in the rabbit. However, it is known that rabbits do not permit development of the parasite to the adult stage (22). In addition, a postmortum examination of the inoculated rabbit eight weeks after the last injection with infective larvae demonstrated that no parasites were present. This suggests that any development of the parasite past the larval stage must have been very limited in nature.

The clones which we have isolated appear to fall into two classes, based on cross hybridization analysis, suggesting that the clones in these two classes are derived from two different mRNA species. It should be possible to identify clones derived from several additional messages, since the larval antiserum is able to precipitate six distinct polypeptides from the products of in vitro translation of adult RNA.

We have characterized a representative from the more immunoreactive class of clones in detail. This clone, designated $\lambda$ RAL-1, contained an insert of $1076 \mathrm{bp}$. Of these $1076 \mathrm{bp}$, 1008 are contained in a single large open reading frame, which is also in frame with the open reading frame of the $\beta$-galactosidase gene found in pUC 13. Together, the combined open reading frames of the $\beta$-galactosidase moity $5^{\prime}$ to the Eco RI site of pUC 13 and the open reading frame of the inserted cDNA predict that this system will produce a fusion protein with a predicted size of 41,454 . This is in good agreement with the 38,000 polypeptide recognized by the adult antiserum in lysates of cultures of $E$. coli that contain the inserted DNA of $\lambda$ RAL-1 sub-cloned into pUC 13 . This large open reading frame is not in frame with the $\beta$-galactosidase gene of $\lambda \mathrm{gt} 11$, however. Although the initial bacteriophage isolate reacts reproducably with both the anti-adult and anti-larval antisera, analysis of induced lysates of this clone using Western blots suggested that a stable fusion product was not being produced. All of these results, when taken together, suggest that the synthesis of the recombinant antigen in the original phage isolate is not under the control of the $\beta$-galactosidase gene of $\lambda \mathrm{gt} 11$. This phenomenon has been noted previously (23), and may been explained by the presence of a fortuitous $E$. coli promoter in the arm of $\lambda \mathrm{gt} 11$ (24).

The inserted DNA of $\lambda$ RAL-1 hybridizes to a single message of 1450 bases in adult $O$. volvulus mRNA. The insert of $\lambda$ RAL-1 is 1076 bp in length and does not appear to contain an

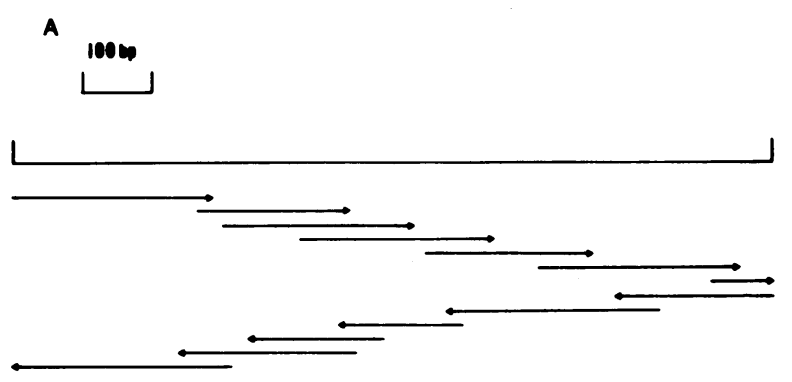

B

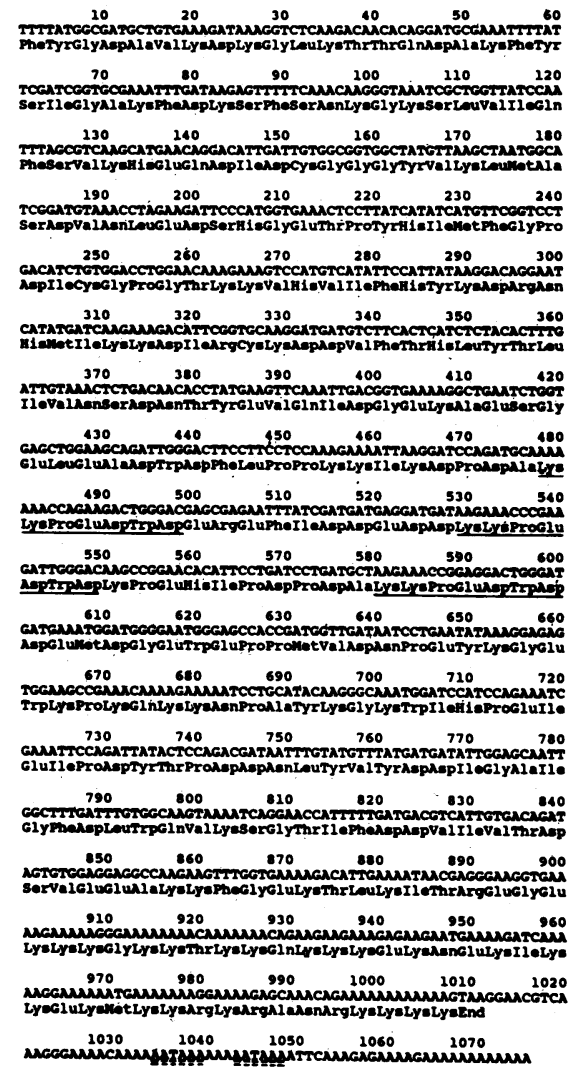

Figure 7. The DNA sequence of the inserted DNA of $\lambda$ RAL-1. The DNA sequence of $\lambda$ RAL-1 was determined as described in Methods. $A$ presents the start point and extent of the sequence obtained from deleted subclones prepared as described in Methods. The complete sequence of the inserted cDNA from $\lambda$ RAL-1 is presented in $B$, along with the predicted amino acid sequence encoded by the long open reading frame discussed in the text. The repeated polypeptide sequence discussed in the text is highlighted by the solid underlining, while the consensus poly $\mathrm{A}$ addition signals are highlighted $\vec{b}$ dotted underlining. 
initiating methionine codon. Thus, it is clear that $\lambda$ RAL-1 is not a full length cDNA clone. Since the sequence contains a single long open reading frame that extends through the presumptive $5^{\prime}$ end of the insert, it is likely that a portion of the coding region found in the native message is missing in $\lambda$ RAL-1. Exactly how much is missing is hard to predict, since the length of the poly $A$ tail on the native messege is not known. However, antibodies which specifically bind to protein prepared from induced cultures of $\lambda$ RAL-1 lysogens recognize a single polypeptide with a molecular weight of 42,000 in extracts of adult worms. Analysis of the long open reading frame encoded by $\lambda$ RAL- 1 suggests that it encodes polypeptide with a molecular weight of 39,130 . If the native antigen is not subjected to posttranslational processing, this suggests that $\lambda$ RAL-1 does contain most of the protein coding sequence found in the native message. More importantly, results using the antilarval antisera described above clearly demonstrate that the recombinant protein produced by $\lambda$ RAL- 1 contains epitopes found in infective larvae. Furthermore, lysates of $\lambda$ RAL- 1 specifically stimulate a cellular immune response, demonstrating that the epitopes which it encodes have relevance in a natural infection. Therefore, even though the insert contained in $\lambda$ RAL-1 is not full length, the recombinant antigen it produces will be useful in studies directed at dissecting the role that the native larval antigen plays in the development of immunity against the infectious form of the parasite.

As mentioned above, a weak hybridization signal is detected when the insert of $\lambda$ RAL- 1 is used to probe Northern blots of $A$. suum RNA. This suggests that a message related to the one that gave rise to $\lambda$ RAL- 1 is found in $A$. suum. This result is not surprising, since results from many previous studies have shown that most of the antigens of different nematode species are not species specific $(25,26)$. Recent studies of antigenic cross reactivity of different parasitic nematodes have suggested that $A$. suum may be the most closely related species to $O$. volvulus by this criterion (27). A more complete characterization of any such related antigen in $\mathrm{A}$. suum will require antisera specific for the recombinant protein produced by $\lambda$ RAL-1.

The fact that two different restriction enzymes produce a single band when a digest of genomic $O$. volvulus DNA is probed with the purified insert from $\lambda$ RAL- 1 suggests that the organization of the gene which encodes $\lambda R A L-1$ is not complex. In particular, the results suggest that there is only one copy of the gene encoding $\lambda R A L-1$ in the genome, and that this copy does not contain any extended introns. Although this is the simplest interpretation, other possibilities, such as the presence of a tandem array of repeated genes homologous to $\lambda$ RAL- 1 are also possible. The isolation and characterization of genomic clones homologous to the insert of $\lambda$ RAL-1 should provide a definitive answer to this question.

One striking characteristic of the polypeptide encoded by the long open reading frame of $\lambda R A L-1$ is the fact that it contains three repeats of the sequence KKPEDWD. These repeats reside in a highly hydrophilic region of the peptide, and are therefore likely to be exposed on the surface of the native antigen. As such, it is likely that this repeated region may represent one of the more highly immunogenic portions of the native antigen (28). Several examples of such repeated sequences have been noted in other parasitic antigens, especially malaria $(29,30)$, and $S$. mansoni $(31)$. It appears that such repeated sequences may be a common feature of antigens iso- lated from many different parasitic species. In the case of other antigens which contain such repeated sequences, the repeated region often encodes the major epitope of the protein $(32,33)$. To discover if this is the case in $\lambda$ RAL-1, epitope mapping utilizing deleted versions of the $\lambda$ RAL -1 insert will be required.

We have taken a first step examining the role that the native antigen encoded by the mRNA that produced $\lambda R A L-1$ may play in natural infections, showing that lysates containing the recombinant antigen stimulate the proliferation of PBMCs from a small number of infected individuals to a greater extent than does a control $\lambda g t 11$ lysate. This is important, since it is believed that the cellular immune system may be particularily important in the development of immunity against helminthic infections (34). Although the responses seen to the recombinant lysate was both reproducible and significant, the overall response was not striking. However, previous studies have demonstrated that the PBMCs of individuals infected with $O$. volvulus show both specific and nonspecific supression of their response to antigens $(35,36)$. The relatively low level of overall response seen to the recombinant lysate is therefore not surprising. It is possible that the nonspecific supression induced by $O$. volvulus infection may have been an asset in this case, by supressing the strong response to $E$. coli antigens one would normally expect to see when using induced lysates as antigens in the blastogenesis assays. This may have allowed the response to the recombinant antigen to be demonstrated. In spite of this fact, it is quite likely that the response of patient cells relative to the control would be improved if purified preparations of the recombinant protein were used in place of crude extracts. Most importantly, these studies demonstrate that it is possible to use such recombinant antigen preparations to begin to examine the role that larval antigens play in natural infections with $O$. volvulus. Such studies will necessarily involve testing both the cellular and antibody responses in a much larger and diverse patient population than has been examined here. It will also be possible to use purified preparations of recombinant antigens in animal model systems which mimic some of the pathological manifastations of human onchocerciasis $(37,38)$, as well as models for protection against infection with the parasite (39). The isolation of $\lambda$ RAL-1 and other clones that express antigens of $O$. volvulus infective larvae will therefore make it possible to examine the role that the antigens of infective larvae may play in the possible development of immunity against onchocerciasis.

\section{Acknowledgments}

We thank D. Rechnitzer for technical support, Drs. P. N. Williams and $J$. Boateng for assistance in obtaining $O$. volvulus material, and Drs. $R$. Blanton, A. Davis, J. Kazura, and N. Lang for helpful comments and suggestions.

Supported by grants from the Edna McConnell Clark Foundation, the John M. and Catherine T. MacArthur Foundation Research Consortium on the Biology of Parasitic Diseases and the U. S. Public Health Service (NIH grants EY-03318 and AI-15351).

\section{References}

1. World Health Organization. 1976. Epidemology of Onchocerciasis. WHO Tech. Rep. Ser. 597:1-94.

2. Kirkwood, B., P. Smith, T. Marshall, and A. Prost. 1983. Relationships between mortality, visual acuity and microfilarial load in the 
area of the onchocerciasis control program. Trans. R. Soc. Trop. Med. Hyg. 77:862-868.

3. Edna McConnell Clark Foundation. 1985. Strategic Plan for Onchocerciasis Research. Edna McConnell Clark Foundation, New York.

4. MacKenzie, C. D., J. F. Williams, B. M. Sisley, M. W. Steward, and J. O'Day. 1985. Variations in host responses and the pathogenesis of human onchocerciasis. Rev. Infect. Dis. 7:802-808.

5. Wong, M. M., M. F. Guest, and M. J. Laviopierre. 1974. Dirofilaria immitis: Fate and immunogenicity of irradiated infective stage larvae in beagles. Exp. Parasitol. 35:465-474.

6. Yates, J. A., and G. I. Higashi. 1985. Brugia malayi: Vaccination of Jirds with ${ }^{60} \mathrm{Cobalt-attenuated} \mathrm{infective} \mathrm{stage} \mathrm{larvae} \mathrm{protects} \mathrm{against}$ homologous challenge. Am. J. Trop. Med. Hyg. 34:1132-1137.

7. Glisin, V., R. Crkvenjakov, and C. Byus. 1974. Ribonucleic acid isolated by cesium chloride centrifugation. Biochemistry. 13:26332637.

8. Schulz-Key, H., E. J. Albiez, and D. W. Buttner. 1977. Isolation of living Adult Onchocerca volvulus from nodules. Tropmed. Parasitol. 28:428-430.

9. Shah, J. S., M. Karam, W. F. Piessens, and D. F. Wirth. 1987. Characterization of an Onchocerca-specific DNA Clone from Onchocerca volvulus. Am. J. Trop. Med. Hyg. 37:376-384.

10. Laemmli, U. K. 1970. Cleavage of structural proteins during the assembly of the head of bacteriophage T4. Nature (Lond.). 227:680-685.

11. Maniatis, T., E. F. Fritsch, and J. Sambrook. 1982. Molecular Cloning: A Laboratory Manual. Cold Spring Harbor Laboratory, Cold Spring Harbor, NY.

12. Gubler, U., and B. J. Hoffman. 1983. A simple and very efficient method for generating cDNA libraries. Gene. 25:263-269.

13. Ham, P. J., and A. J. Banya. 1984. The effect of experimental Onchocerca infections on the fecundity and oviposition of laboratory reared Simulium sp. (Diptera: Simuliidae). Tropenmed. Parasitol. 35:61-66.

14. Huynh, T. V., R. A. Young, and R. W. Davis. 1984. Constructing and screening cDNA libraries in $\lambda g t 10$ and $\lambda g t 11$. In Cloning Techniques: A Practical Approach. D. Glover, editor. IRL Press, Oxford.

15. Vogelstein, B., and D. Gillespie. 1979. Preparative and analytical purification of DNA from agarose. Proc. Natl. Acad. Sci. USA. 76:615-619.

16. Schreier, P. H., and R. Cortese. 1979. A fast and simple method for sequencing DNA Cloned in the single-stranded bacteriophage M13. J. Mol. Biol. 129:169-172.

17. Sanger, F., S. Nicklen, and A. R. Coulson. 1977. DNA sequencing with chain terminating inhibitors. Proc. Natl. Acad. Sci. USA. 74:5463-5467.

18. Towbin, H., T. Staehelin, and J. Gordon. 1979. Electrophoretic Transfer of proteins from polyacrylamide gels to nitrocellulose sheets: procedure and some applications. Proc. Natl. Acad. Sci. USA. 76:4350-4354.

19. Southern, E. M. 1975. Detection of specific sequences among DNA fragments separated by gel electrophoresis. J. Mol. Biol. 98:503-517.

20. Hopp, T. P., and K. R. Woods. 1981. Prediction of Protein Antigenic Determinants from Amino Acid Sequences. Proc. Natl. Acad. Sci. USA. 78:3824-3828.

21. Wickens, M., and P. Stephenson. 1984. Role of the conserved AAUAAA sequence: four AAUAAA point mutants prevent messenger RNA 3' end formation. Science (Wash. DC). 226:1045-1051.
22. Kozek, W. J., and H. F. Marroquin. 1982. Attempts to establish Onchocerca volvulus infection in primates and small laboratory animals. Acta Trop. 39:317-324.

23. Dame, J. B., J. L. Williams, T. F. McCutchan, J. L. Weber, R. A. Wirtz, W. T. Hockmeyer, W. L. Maloy, J. D. Haynes, I. Schneider, D. Roberts, G. S. Sanders, E. P. Reddy, C. L. Diggs, and L. H. Miller. 1984. Structure of the gene encoding the immunodominant surface antigen on the sporozoite of the human malaria parasite Plasmodium falciparum. Science (Wash. DC). 225:593-599.

24. Chirala, S. S. 1986. The nucleotide sequence of the Lac operon and phage junction in lambda gt 11. Nucleic Acids Res. 14:5935.

25. Ambroise-Thomas, P. 1974. Immunological diagnosis of human filariasis: present possibilities, difficulties and limitations. Acta Trop. 38:108-128.

26. Higashi, G. I. 1984. Immunodiagnostic tests for protozoan and helminthic infections. Diagno. Immunol. 2:2-18.

27. Nogami, S., Y. Hayashi, M. Tanaka, M. Korenaga, I. Tada, and H. Tanaka. 1986. Antigenic similarity of Onchocerca volvulus to other helminths examined by monoclonal antibodies against $O$. volvulus. Jpn. J. Exp. Med. 56:177-183.

28. Berzofsky, J. A. 1985. Intrinsic and extrinsic factors in protein antigen structure. Science (Wash. DC). 229:932-940.

29. Enea, V., J. Ellis, F. Zavala, D. E. Arnot, A. Asavanich, A. Masuda, I. Quakyi, and R. S. Nussenzweig. 1984. DNA cloning of Plasmodium falciparum circumsporozoite gene: amino acid sequence of repetitive epitope. Science (Wash. DC). 225:628-630.

30. Ravetch, J. V., J. Kochan, and M. Perkins. 1985. Isolation of the gene for a glycophorin-binding protein implicated in erythrocyte invasion by a malaria parasite. Science (Wash. DC). 227:1593-1597.

31. Bobek, L., D. M. Rekosh, H. van Keulen, and P. T. LoVerde. 1986. Characterization of a female-specific cDNA derived from a developmentally regulated mRNA in the human blood fluke Schistosoma mansoni. Proc. Natl. Acad. Sci. USA. 83:5544-5548.

32. Ballou, W. R., J. Rothbard, R. A. Wirtz, D. M. Gordon, J. S. Williams, R. W. Gore, I. Schneider, M. R. Hollingdale, R. L. Beaudoin, W. L. Maloy, L. H. Miller, and W. T. Hockmeyer. 1985. Immunogenicity of synthetic peptides from circumsporozoite protein of Plasmodium falciparum. Science (Wash. DC). 228:996-999.

33. Collins, W. E., R. F. Anders, M. Pappaioanou, G. H. Campbell, G. V. Brown, D. J. Kemp, R. L. Coppel, J. C. Skinner, P. M. Andrysiak, J. M. Favaloro, L. M. Corcoran, J. R. Broderson, G. F. Mitchell, and C. C. Campbell. 1986. Immunization of aotus monkeys with recombinant proteins of an erythrocyte surface antigen of Plasmodium falciparum. Nature (Lond.). 323:259-262.

34. Lal, R. B., T. J. Lynch, and T. B. Nutman. 1987. Brugia malayi antigens associated with lymphocyte activation in filariasis. J. Immunol. 139:1652-1657.

35. Ngu, J. L. 1978. Immunological Studies on Onchocerciasis. Acta Trop. 35:269-279.

36. Greene, B. M., M. M. Fanning, and J. J. Ellner. 1983. Non-specific supression of antigen-induced lymphocyte blastogenesis in $\mathrm{On-}$ chocerca volvulus infection in man. Clin. Exp. Immunol. 52:259-265.

37. Donnelly, J. J., J. H. Rockey, A. E. Bianco, and E. J. L. Soulsby. 1984. Ocular immunopathologic findings of experimental onchocerciasis. Arch. Ophthalmol. 102:628-634.

38. Donnelly, J. J., H. R. Taylor, E. Young, M. Khatami, J. B. Lok, and J. H. Rockey. 1986. Experimental ocular onchocerciasis in cynomolgus monkeys. Inv. Ophthalmol. Vis. Sci. 27:492-499.

39. Greene, B. M. 1987. Primate model for onchocerciasis research. In Filariasis, Ciba Foundation Symposium 127. D. Evered and S. Clark, editors. John Wiley and Sons, New York. 236-240. 